\title{
Hourglass Epidermoid Inclusion Cyst: An Unusual Clinical Presentation
}

\author{
Vijay Naraynsingh, MBBS, FRCS, Seetharaman Hariharan, MD, FCCM, \\ Dilip Dan, MBBS, FACS, and Patrick Harnarayan, MBBS, FRCS*
}

The authors have indicated no significant interest with commercial supporters.

\begin{abstract}
A lthough hourglass epidermoid inclusion cysts have been reported in the cranial region, they have not been reported elsewhere in the body. We report a case of an epidermoid inclusion cyst that was excised completely.
\end{abstract}

\section{Case Report}

An obese 60-year-old woman presented with a 2-year history of a slowly enlarging, painless lump in the hip region. On examination, it was found to be a typical sebaceous cyst, $2.5 \mathrm{~cm}$ in diameter, attached to the skin with a punctum at the apex over the greater trochanter laterally. There was no sign of scarring or inflammation.

Under local anesthesia (1\% lidocaine with epinephrine), an elliptical incision including the punctum was made. The dissection was uneventful, with the plane around the cyst well defined and with no obvious fibrous tissue. On reaching around the deep surface of the cyst, there appeared to be tethering. Careful dissection and exposure revealed that the cyst continued on, beyond a narrow neck, to another "lobe" that was approximately the same size as the superficial portion (Figure 1). The cyst was removed completely. On cut section, the two portions, each $2.5 \mathrm{~cm}$ in diameter, were in communication through a neck $2 \mathrm{~mm}$ in diameter (Figure 2).

\section{Discussion}

Epidermoid inclusion cysts do not always need surgical treatment but are commonly excised for cosmetic reasons and complications. Surgical approaches are of two categories: traditional wide excision and minimal excision with punch biopsy. ${ }^{1}$ Although minimal excision has been advocated, it is unclear whether this technique is useful for larger cysts and prevents recurrence. ${ }^{2}$

There have been no randomized controlled trials published that compare the most common surgical techniques for treatment of sebaceous cysts. Only one small randomized study involving 60 patients compared traditional wide excision with punch biopsy. ${ }^{3}$ Although this study found punch biopsy to be less time consuming and capable of offering a superior cosmetic result, it is doubtful that larger cysts could be removed using this technique.

There have been two reports of hourglass epidermoid cysts in the cranial cavity, but there is no report of this anatomical variation in other parts of the body. ${ }^{4,5}$

In sebaceous cyst surgery, the cyst may rupture, and later recurrence is possible. If the assumption is made that the deep surface of a sebaceous cyst is a smooth convexity, blunt dissection of this area could result in rupture and recurrence if the cyst has an

*All authors are affiliated with Department of Clinical Surgical Sciences, University of the West Indies, St. Augustine, Trinidad, West Indies 


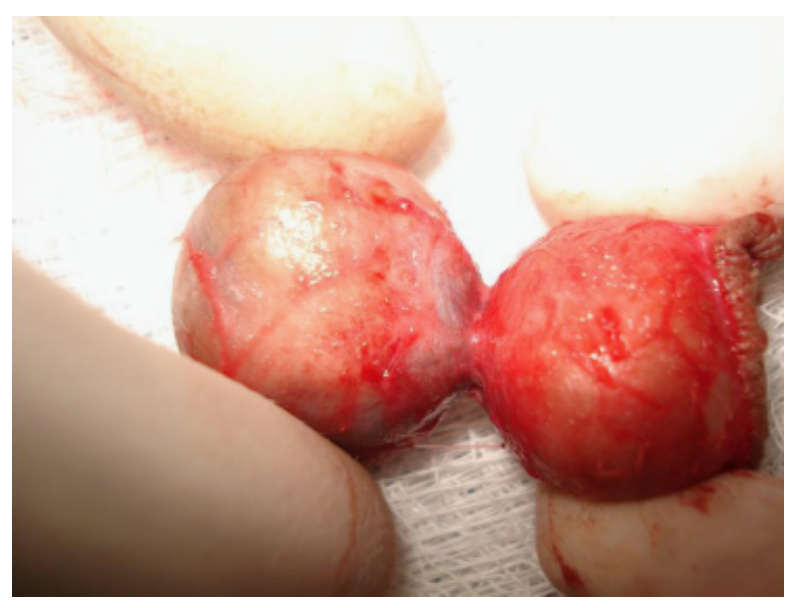

Figure 1. Gross appearance of the "hourglass" sebaceous cyst.

hourglass deformity. This abnormality could be difficult to recognize if the field of dissection is bloody, the incision too small, or the lighting inadequate - conditions that often apply, because this procedure is usually regarded as minor and may be performed in less-than-ideal settings and by junior or inexperienced staff.

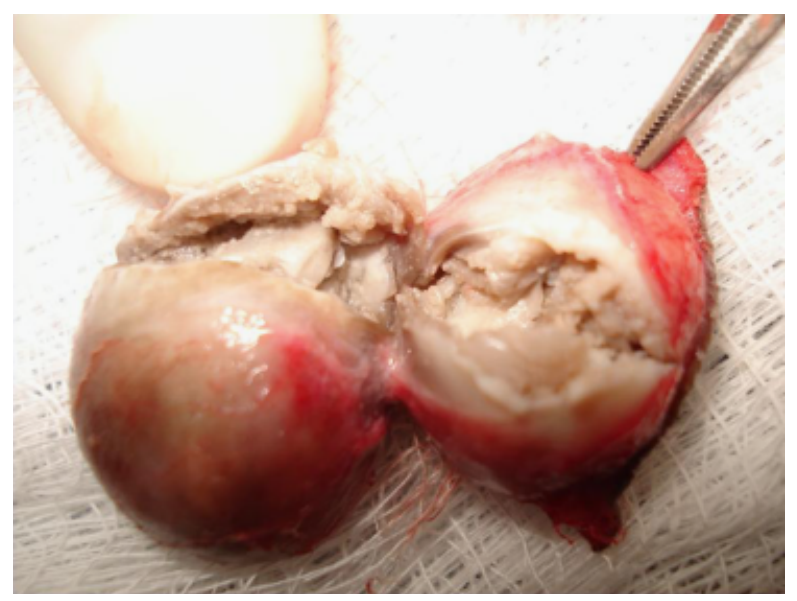

Figure 2. Hourglass sebaceous cyst after being cut open.
In this case, there was no scarring, fibrosis, or abnormality of any part of the cyst wall or surrounding tissues, but the wall of a sebaceous cyst varies in thickness. Because this cyst was near the greater trochanter in an obese patient, one could postulate that sustained, repeated pressure from sitting could produce a small "diverticulum" at the weakest point in the cyst wall. This might then enlarge progressively as the sebum accumulates, producing an hourglass appearance.

If this is true, it may offer a simple anatomical explanation for the common occurrences of rupture at surgery and recurrence thereafter if an hourglass deformity or a much smaller "diverticulum" projects from the otherwise smooth sphere of a sebaceous cyst, resulting in incomplete excision.

\section{References}

1. Moore RB, Fagan EB, Hulkower S, et al. What's the best treatment for sebaceous cysts? J Fam Pract 2007;56:315-6.

2. Zuber TJ. Minimal excision technique for epidermoid (sebaceous) cysts. Am Fam Phys 2002;65:1409-12, 1417-8, 1420.

3. Lee HE, Yang $\mathrm{CH}$, Chen $\mathrm{CH}$, et al. Comparison of the surgical outcomes of punch incision and elliptical excision in treating epidermal inclusion cysts: a prospective, randomized study. Dermatol Surg 2006;32:520-5.

4. Nagayama M, Shinohara Y, Takeoka T, et al. Intracranial paramedian hourglass-shaped dermoid associated with hereditary steatocystoma multiplex. Eur Neurol 1992;32:260-3.

5. Maiuri F, Donzelli R, Spaziante R, et al. Paramedian hourglass epidermoid cysts extending in the middle and posterior cranial fossa. J Neurosurg Sci 1986;30:187-96.

Address correspondence and reprint requests to: Seetharaman Hariharan, MD, FCCM, Senior Lecturer, Department of Clinical Surgical Sciences, Faculty of Medical Sciences, The University of the West Indies, Eric Williams Medical Sciences Complex, Mount Hope, Trinidad, West Indies, or e-mail: uwi.hariharan@gmail.com 